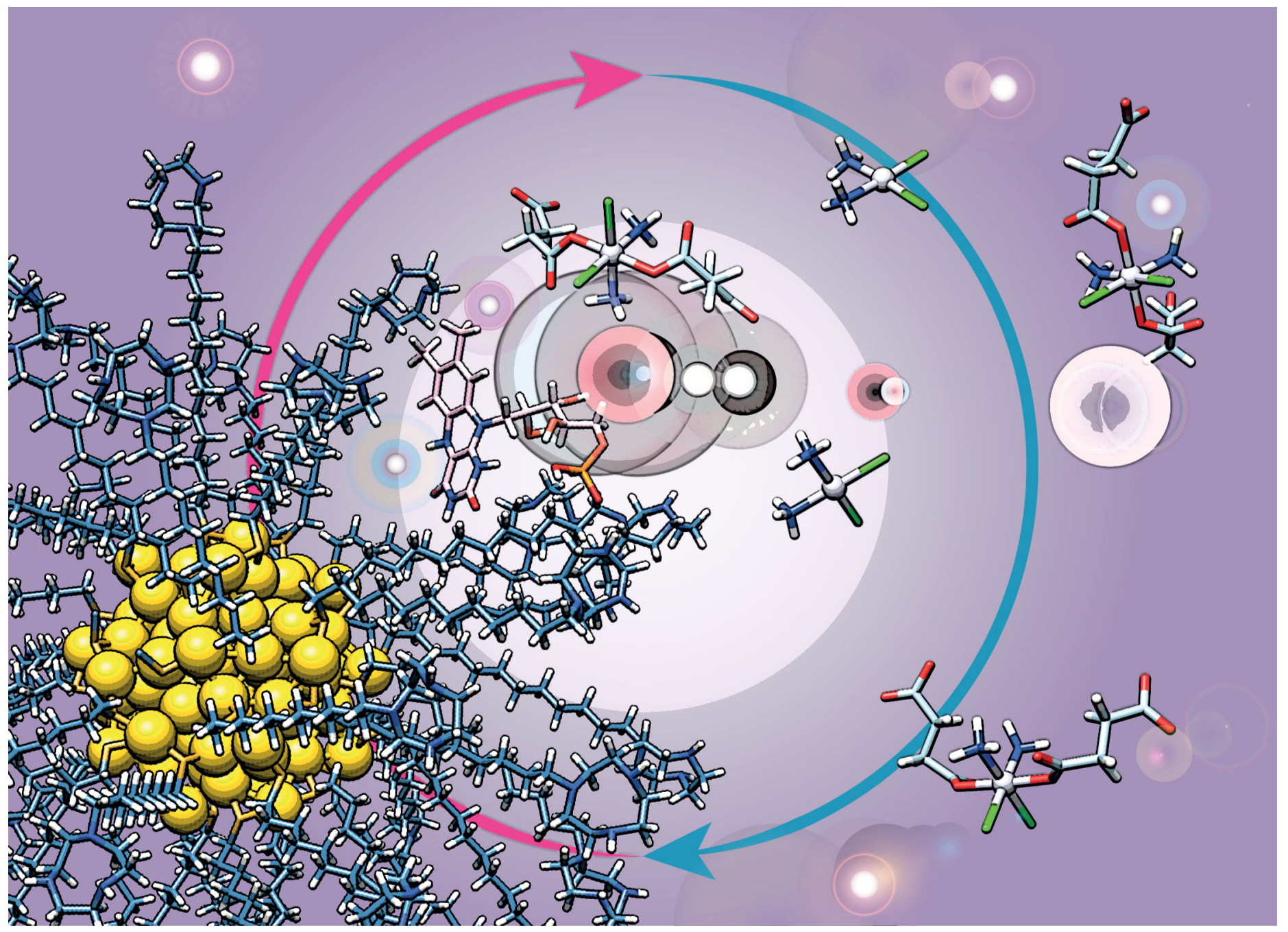

Showcasing research from Professor Salassa's and Mancin's laboratory, Donostia International Physics Center, Donostia/San Sebastián, Spain and Department of Chemical Sciences, University of Padua, Padua, Italy.

Toward supramolecular nanozymes for the photocatalytic activation of $\mathrm{Pt}(\mathrm{IV})$ anticancer prodrugs

Au nanoparticles decorated with thiol ligands bearing a TACN headgroup encapsulate FMN to afford a supramolecular nanozyme capable of photocatalyzing the reductive activation of a $\mathrm{Pt}(\mathrm{IV})$ prodrug to cisplatin. 
Check for updates

Cite this: Chem. Commun., 2020, 56, 10461

Received 14th May 2020,

Accepted 28th July 2020

DOI: $10.1039 / \mathrm{d} 0 \mathrm{cc} 03450 \mathrm{a}$

rsc.li/chemcomm

\section{Toward supramolecular nanozymes for the photocatalytic activation of Pt(Iv) anticancer prodrugs $\dagger$}

\author{
Laura F. Mazzei, ${ }^{\text {abc }}$ Álvaro Martínez, ${ }^{\star a}$ Lucia Trevisan, (iD c Daniele Rosa-Gastaldo, (ID c \\ Aitziber L. Cortajarena, (ID bd Fabrizio Mancin (D) *c and Luca Salassa (D) *ade
}

\begin{abstract}
A supramolecular nanozyme for the photocatalytic conversion of a $\mathrm{Pt}(\mathrm{IV})$ anticancer complex to cisplatin is described herein. We employed $1.9 \mathrm{~nm}$ Au nanoparticles decorated with thiol ligands bearing a TACN (1,4,7-triazacyclononane) headgroup to encapsulate FMN (riboflavin-5' -phosphate). In the presence of an electron donor, flavin-loaded nanoparticles photocatalyzed the reductive activation of the prodrug cis, cis, trans- $\left[\mathrm{Pt}\left(\mathrm{NH}_{3}\right)_{2}\left(\mathrm{Cl}_{2}\right)\left(\mathrm{O}_{2} \mathrm{CCH}_{2} \mathrm{CH}_{2} \mathrm{COOH}\right)_{2}\right]$ to cisplatin, achieving turnover frequency values of $7.4 \mathrm{~min}^{-1}$.
\end{abstract}

Bioorthogonal nanozymes are nm-sized catalysts capable of performing artificial reactions in biological media. ${ }^{1}$ To date, nanozymes have been fabricated by entrapping metallic nanoparticles (e.g. $\mathrm{Pd}, \mathrm{Au}, \mathrm{Cu}$ ) into polymer matrixes, ${ }^{2-4}$ by decorating nanometric $\mathrm{Au}$ scaffolds with catalytic units ${ }^{5}$ or by encapsulating organometallic catalysts (e.g. Ru, Pd complexes) in the coating ligand shell. ${ }^{6}$ Especially in the latter case, the nanostructure is fundamental to improve the solubility of the embedded catalyst and to protect it from poisoning reactions that easily occur in biological environments. ${ }^{7}$ Besides, proper design of the nanozyme enables controlling the localization of the molecular catalyst in specific cellular compartments ${ }^{3,8}$ or tissues $^{9}$ and triggering their catalytic activity upon external stimuli. ${ }^{6}$ To the best of our knowledge, bioorthogonal nanozymes developed so far catalyze the deprotection of caged organic imaging and therapeutic agents or promote the in situ synthesis of drug derivatives. ${ }^{1,4,10}$

\footnotetext{
${ }^{a}$ Donostia International Physics Center, Paseo Manuel de Lardizabal 4, Donostia, 20018, Spain. E-mail: lsalassa@dipc.org

${ }^{b}$ CIC biomaGUNE, Basque Research Technology Alliance, BRTA, Parque Tecnológico de San Sebastián, Paseo Miramón 194, 20014 Donostia/San Sebastián, Spain ${ }^{c}$ Dipartimento di Scienze Chimiche, Università di Padova, via Marzolo 1, Padova, 35131, Italy. E-mail: fabrizio.mancin@unipd.it

${ }^{d}$ Ikerbasque, Basque Foundation for Science, Bilbao, 48011, Spain

${ }^{e}$ Kimika Fakultatea, Euskal Herriko Unibertsitatea, UPV/EHU, Donostia, 20080, Spain

$\dagger$ Electronic supplementary information (ESI) available. See DOI: 10.1039/ docc03450a
}

We recently reported a novel type of bioorthogonal photocatalytic reactions in which flavins (e.g. riboflavin, FMN and FAD) and certain flavoproteins transform Pt(Iv) precursors into the clinically approved anticancer drugs cisplatin and carboplatin in biological media (Fig. 1A). ${ }^{11-13}$ As rarely observed in catalysis, ${ }^{14,15}$ metal complexes act as substrates rather than catalysts in these unconventional processes. Mechanistic studies highlighted that light excitation of flavins in the presence
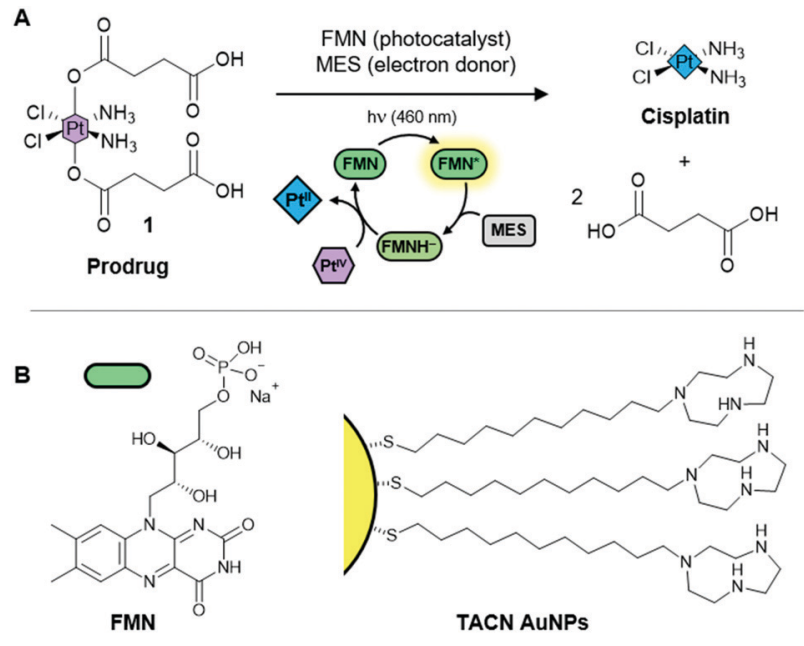

C

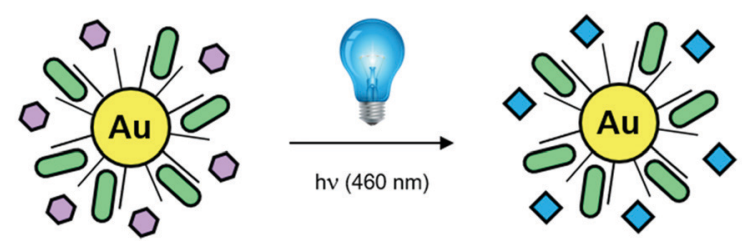

Fig. 1 (A) Photocatalytic activation scheme of the cisplatin prodrug cis,cis,trans- $\left[\mathrm{Pt}\left(\mathrm{NH}_{3}\right)_{2}\left(\mathrm{Cl}_{2}\right)\left(\mathrm{O}_{2} \mathrm{CCH}_{2} \mathrm{CH}_{2} \mathrm{COOH}\right)_{2}\right]$ (1); (B) photocatalyst (FMN) and $\mathrm{Au}$ nanoparticles decorated with a $\mathrm{C}_{11}$-thiol bearing a 1,4,7-triazacyclononane headgroup (TACN AuNPs); (C) schematic representation of the nanozyme developed in this work. 
of an electron donor (e.g. zwitterionic buffers or NADH) affords the doubly-reduced flavin form (Fig. 1A) which is the active catalyst prompting the bioorthogonal substrate conversion. ${ }^{11,13}$ In vitro experiments also demonstrated that this activation strategy hold promise for its capacity to deliver lethal concentrations of Pt drugs with minimal light doses. ${ }^{11,16}$

The relevance of $\mathrm{Pt}(\mathrm{Iv})$ complexes in photochemotherapy research is proved by the numerous photoactivatable prodrugs and activation approaches developed in recent years. ${ }^{17-20}$

Motivated by these results and the progress in the design of nanomaterials with enzyme-like features, we report herein our first steps in the implementation of photocatalysis towards Pt substrates by nanozymes. To such aim, we selected FMN, $1.9 \mathrm{~nm} \mathrm{Au}$ nanoparticles decorated with a $\mathrm{C}_{11}$-thiol bearing a 1,4,7-triazacyclononane headgroup (TACN AuNPs), and the complex cis, cis,trans- $\left[\mathrm{Pt}\left(\mathrm{NH}_{3}\right)_{2}\left(\mathrm{Cl}_{2}\right)\left(\mathrm{O}_{2} \mathrm{CCH}_{2} \mathrm{CH}_{2} \mathrm{COOH}\right)_{2}\right]$ (1) as photocatalyst, nanozyme scaffold, and substrate, respectively (Fig. 1A and B; Fig. S1 and S2, ESI $\dagger$ ).

We opted for spherical nanoparticles with a Au core smaller than $2 \mathrm{~nm}$ to have a nanoplatform size in the biomolecular scale and to minimize light absorption and scattering by the nanoparticles due to the surface plasmon band, whose intensity depends on the nanoparticle size. ${ }^{21}$ Previously, Rotello and co-workers used similar AuNPs for the controlled photodelivery of anticancer drugs using UV-A light. ${ }^{22}$ NMR sensing studies on TACN AuNPs closely related to the ones used in this work showed that FMN strongly binds to the thiol monolayer via electrostatic and hydrophobic interactions. ${ }^{23}$ When in the protonated form $\left(\mathrm{TACN}_{\mathrm{NH}} \mathrm{p} K_{\mathrm{a}} 10.4,6.8,2.4\right)^{24}$ or bound to $\mathrm{Zn}^{2+}$, the positively charged TACN group can interact with the negatively charged phosphate of the FMN ribityl sidechain, while the hydrophobic environment provided by the $\mathrm{C}_{11}$ alkyl spacer of the ligand can accommodate the aromatic isoalloxazine ring system of FMN. Likewise, 1 is also capable of electrostatically binding the TACN headgroups, since the succinato ligands of the complex are deprotonated at $\mathrm{pH}$ above $6 .^{25}$ Therefore, TACN AuNPs are valuable candidates to operate as supramolecular nanoscaffolds that favor co-localization of FMN and 1, ultimately affording a nanozyme for the photocatalytic conversion of a $\mathrm{Pt}$ (Iv) prodrug into cisplatin (Fig. 1C).

To test this hypothesis and assemble the nanozyme, we firstly investigated the loading of FMN onto TACN AuNPs (100 $\mu \mathrm{M}$ of TACN ligand) in MES buffer ( $5 \mathrm{mM}, \mathrm{pH} 6)$ by fluorescence quenching measurements. These experiments were performed in the absence and presence of 1 equivalent of $\mathrm{Zn}\left(\mathrm{NO}_{3}\right)_{2}(100 \mu \mathrm{M})$, that is with the amino groups of TACN positively charged due to protonation (FMN@TACN AuNPs) or coordination of $\mathrm{Zn}^{2+}$ (FMN@Zn-TACN AuNPs) respectively. MES was selected since it acts as electron donor in the $\mathrm{Pt}(\mathrm{IV}) \rightarrow \mathrm{Pt}(\mathrm{II})$ catalytic conversion and protects flavins from photodecomposition. ${ }^{11,26}$ Upon interaction with TACN AuNPs, FMN fluorescence emission $\left(\lambda_{\mathrm{em}}=527 \mathrm{~nm}\right)$ was quenched by the Au core of the nanoparticles, enabling determination of the FMN concentration at which the nanoscaffold binding sites are saturated. After correcting FMN emission intensity for inner filter effect ${ }^{27}$ (Fig. S3, ESI $\dagger$ ), we found saturation concentrations

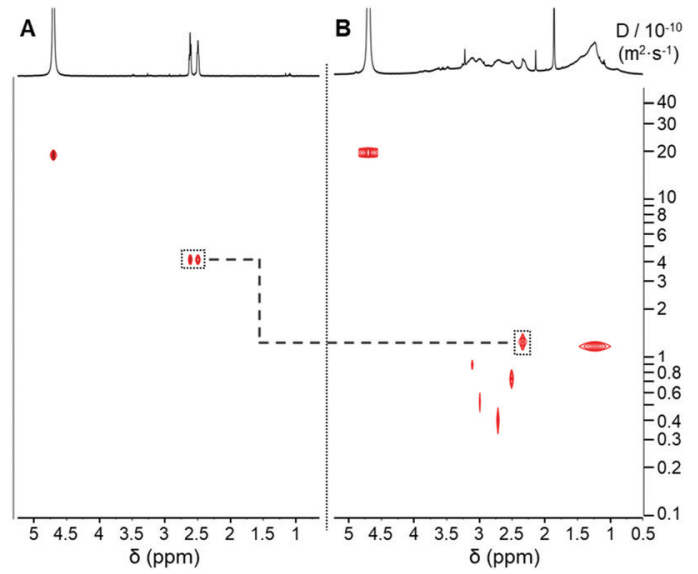

Fig. 2 DOSY spectra of 1 in the absence (A) or presence (B) of TACN AuNPs in $\mathrm{D}_{2} \mathrm{O}$ at $28{ }^{\circ} \mathrm{C}$. [1] $=500 \mu \mathrm{M},[\mathrm{TACN}]_{\text {AuNPs }}=2 \mathrm{mM}$

to be in the 50-60 $\mu \mathrm{M}$ range for both types of TACN AuNPs. Furthermore, DOSY and NOE-pumping NMR experiments confirmed the anticipated interaction between TACN AuNPs and 1 (Fig. 2 and Fig. S4, ESI $\dagger$ ), including under the condition employed in catalysis tests (vide infra). As expected for such a scenario, signals of $\mathbf{1}$ undergo a relevant broadening in the presence of TACN AuNPs (Fig. S4, ESI $\dagger$ ) and the diffusion rate of 1 determined by DOSY decreased up to $c a$. 4 times in the presence of the nanoparticles with respect to the free complex $\left(1.26 \times 10^{-10}\right.$ vs. $\left.4.15 \times 10^{-10} \mathrm{~m}^{2} \mathrm{~s}^{-1}\right)$. NOE pumping unequivocally demonstrated the fast exchange of nanoparticle-bound $\mathbf{1}$ in the NMR time scale. In fact, magnetization transfer from the AuNPs to the metal complex can only be observed in the case of close $(<4 \AA$ ) and sufficiently prolonged contacts.

For testing the catalytic performance of the nanozyme, we hence loaded the nanoparticles with $25 \mu \mathrm{M}$ FMN, a concentration considerably below the saturation limit to avoid the presence of free catalyst in solution during the reaction. The concentration of substrate 1 was fixed at $500 \mu \mathrm{M}$ to allow monitoring the photocatalysis progression by ${ }^{1} \mathrm{H}$-NMR. Importantly, fluorescence control experiments showed that FMN is not significantly displaced by $\mathbf{1}$ at such concentrations (Fig. S5, $\mathrm{ESI} \dagger$ ), confirming the higher affinity of the TACN AuNPs for amphiphilic guests as FMN than for hydrophilic guests as 1. . $^{28,29}$

Under these conditions, we evaluated the capacity of the flavonanozymes to photocatalytically activate $\mathbf{1}$ upon irradiation with blue light (460 nm, $5.30 \mathrm{~mW} \mathrm{~cm}^{-2}$ ) and compared their efficiency with $25 \mu \mathrm{M}$ of free FMN (Fig. 3 and Fig. S6, ESI $\dagger$ ).

Substrate conversion was monitored by quantifying the release of succinato ligands by ${ }^{1} \mathrm{H}-\mathrm{NMR}$ as a function of the irradiation time. Previous work demonstrated that this process corresponded to the generation of cisplatin. ${ }^{16}$

FMN@TACN and FMN@Zn-TACN AuNPs displayed an almost identical behavior in terms of catalytic activity, reaching $80 \%$ conversion already within $7 \mathrm{~min}$ and a plateau at $90 \%$ conversion after $30 \mathrm{~min}$ of light irradiation. These data corresponded to a turnover number (TON) of 18 and turnover frequency (TOF) of $7.4 \mathrm{~min}^{-1}$ (calculated in the range 15-45\% 


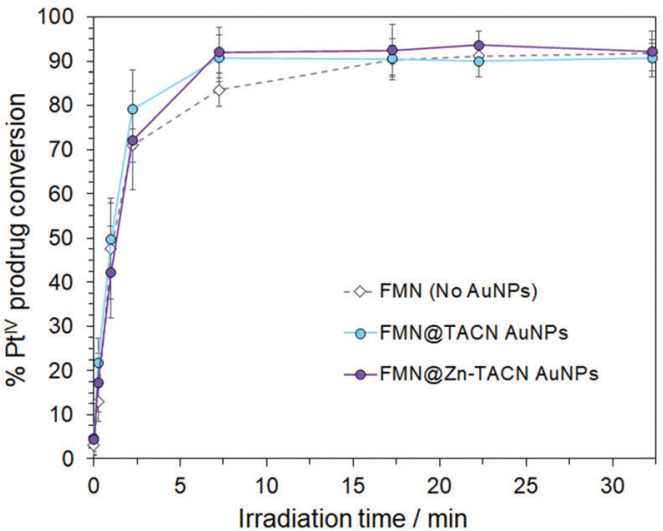

Fig. 3 Photocatalytic activation of 1 by FMN, FMN@TACN AuNPs and FMN@Zn-TACN AuNPs. Experimental conditions: [FMN] $=25 \mu \mathrm{M},[1]=500 \mu \mathrm{M}$, $[\mathrm{MES}]_{\mathrm{pH} 6}=5 \mathrm{mM},[\mathrm{TACN}]_{\mathrm{AuNPs}}=0 \mu \mathrm{M}$ or $100 \mu \mathrm{M},\left[\mathrm{Zn}\left(\mathrm{NO}_{3}\right)_{2}\right]=0 \mu \mathrm{M}$ or $100 \mu \mathrm{M}, h \nu 460 \mathrm{~nm}\left(5.30 \mathrm{~mW} \mathrm{~cm}{ }^{-2}\right)$. Each point corresponds to an average of a triplicate of triplicate independent measurements.

conversion). Similar TON and TOF values were obtained for free FMN under the same conditions. Furthermore, we investigated the stability of the nanozymes under light irradiation to discard leaking of FMN from the AuNPs during catalysis (Fig. 4). In the presence of TACN AuNPs, the fluorescence emission of FMN remained quenched throughout the whole light-irradiation time window, thus demonstrating that the catalyst was associated to the nanozyme scaffold during such period. In addition, the emission intensity of FMN@TACN AuNPs underwent a further slight decrease over time due to FMN photodegradation, ${ }^{11}$ with almost complete depletion after $10 \mathrm{~min}$. However, the process occurred at a lower rate than for free FMN (Fig. 4, inset plot), suggesting a moderate protecting effect of the AuNPs towards FMN photobleaching. We speculate that such increase in stability positively contributed to the catalytic activity of the nanozymes. Inactivation of the catalyst after $10 \mathrm{~min}$ of light irradiation was

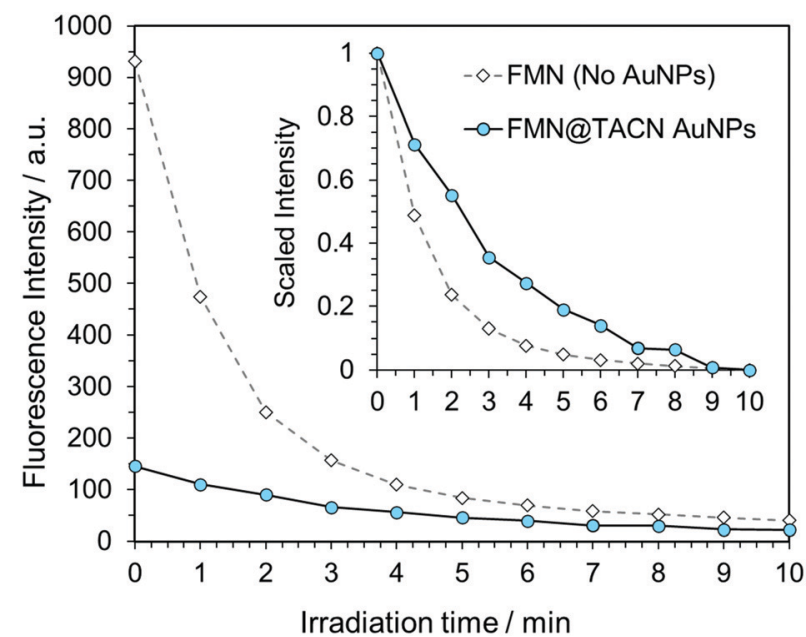

Fig. 4 Photobleaching of FMN@TACN AuNPs and free FMN. Experimental conditions: $[\mathrm{FMN}]=25 \mu \mathrm{M},[1]=500 \mu \mathrm{M},[\mathrm{MES}]_{\mathrm{pH} 6}=5 \mathrm{mM},[\mathrm{TACN}]_{\mathrm{AuNPs}}=$ $0 \mu \mathrm{M}$ or $100 \mu \mathrm{M}, h \nu 460 \mathrm{~nm}\left(5.30 \mathrm{~mW} \mathrm{~cm}^{-2}\right)$. consistent with the plateau observed in the substrate conversion curves of Fig. 3.

As previously reported, FMN alone cannot catalytically reduce 1 in the absence of light, nor under light irradiation without electron donor. ${ }^{13}$ Controls showed that TACN AuNPs only prompted a $15 \%$ substrate conversion in the dark, both with and without FMN loaded (Fig. S7 and S8, ESI $\dagger$ ). Conversely, the nanoscaffold (TACN AuNPs) displayed certain catalytic activity upon light irradiation, reaching $50-70 \%$ conversion in $30 \mathrm{~min}$. Importantly, the reaction efficiency was significantly lower in terms of TOF value $\left(<0.9 \mathrm{~min}^{-1}\right)$ compared to FMN@TACN AuNPs and free FMN (Fig. S9 and S10, ESI $\dagger$ ). High TOF values under light irradiation are valuable for application in photochemotherapy since they permit rapid conversion of $\mathrm{Pt}(\mathrm{Iv})$ prodrugs with low light doses.

Although further investigation is required, the background catalytic activity of TACN AuNPs towards the photoreduction of 1 could be reasonably ascribed to an excited-state electron transfer process promoted by the nanomaterial, considering that its absorption tail extends over $800 \mathrm{~nm}$ (Fig. S2, ESI $\dagger$ ).

Undoubtedly, the roadmap to bioorthogonal nanozymes for the catalytic activation of Pt drugs in complex biological environments still demands a more refined design than the one described in this work. Use of these systems in vitro and in vivo requires that their robustness increases without reducing the catalytic activity. For instance, inclusion of PEGylated ligands in the nanoparticle monolayer will likely improve the colloidal stability and biocompatibility of FMN@TACN and FMN@Zn$\mathrm{TACN}^{30}{ }^{30}$ while providing enhanced protection of the FMN catalyst from the chemical and biological components present in cells and tissues. While these key aspects are worth exploring in the future, the results described here highlight that TACN AuNPs are suitable components to develop supramolecular nanomaterials capable to carry out unconventional flavinmediated catalytic reactions using $\mathrm{Pt}(\mathrm{Iv})$ prodrugs as substrates. Besides extending the repertoire of reactions catalyzed by TACN-based nanoparticles, ${ }^{31-33}$ this work holds promise as strategy to control spatio-temporally the effects of Pt anticancer drugs via light activation and catalytic amplification.

We acknowledge financial support from the Spanish State Research Agency for the grants CTQ2016-80844-R, BIO201677367-R, PID2019-111649RB-I00 and PCI2018-092984. This work was performed under the Severo Ochoa Centres of Excellence and Maria de Maeztu Units of Excellence Program from the Spanish State Research Agency - Grant No. CEX2018-000867-S (DIPC) and MDM-2017-0720 (CIC biomaGUNE). We also thank Fondazione Cariparo (Ricerca Scientifica di Eccellenza Grant "SELECT"). Á. M. has received funding from the European Union's Horizon 2020 Research and Innovation Programme under Marie SklodowskaCurie Actions grant no. 793702. We thank the Spanish MultiMetDrugs network (RED2018-102471-T) for fruitful discussion.

\section{Conflicts of interest}

There are no conflicts to declare. 


\section{Notes and references}

1 X. Zhang, R. Huang, S. Gopalakrishnan, R. Cao-Milán and V. M. Rotello, Trends Chem., 2019, 1, 90-98.

2 R. M. Yusop, A. Unciti-Broceta, E. M. V. Johansson, R. M. SánchezMartín and M. Bradley, Nat. Chem., 2011, 3, 239-243.

3 A. M. Pérez-López, B. Rubio-Ruiz, V. Sebastián, L. Hamilton, C. Adam, T. L. Bray, S. Irusta, P. M. Brennan, G. C. Lloyd-Jones, D. Sieger, J. Santamaría and A. Unciti-Broceta, Angew. Chem., Int. Ed., 2017, 56, 12548-12552.

4 J. Clavadetscher, S. Hoffmann, A. Lilienkampf, L. Mackay, R. M. Yusop, S. A. Rider, J. J. Mullins and M. Bradley, Angew. Chem., Int. Ed., 2016, 55, 15662-15666.

5 F. Mancin, L. J. Prins, P. Pengo, L. Pasquato, P. Tecilla and P. Scrimin, Molecules, 2016, 21, 1014.

6 G. Y. Tonga, Y. Jeong, B. Duncan, T. Mizuhara, R. Mout, R. Das, S. T. Kim, Y. C. Yeh, B. Yan, S. Hou and V. M. Rotello, Nat. Chem., 2015, 7, 597-603.

7 H. Wei and E. Wang, Chem. Soc. Rev., 2013, 42, 6060-6093.

8 A. Gupta, R. Das, G. Yesilbag Tonga, T. Mizuhara and V. M. Rotello, ACS Nano, 2018, 12, 89-94.

9 J. T. Weiss, J. C. Dawson, K. G. Macleod, W. Rybski, C. Fraser, C. Torres-Sánchez, E. E. Patton, M. Bradley, N. O. Carragher and A. Unciti-Broceta, Nat. Commun., 2014, 5, 3277.

10 S. Alonso-de Castro, A. Terenzi, J. Gurruchaga-Pereda and L. Salassa, Chem. - Eur. J., 2019, 25, 6651-6660.

11 S. Alonso-de Castro, E. Ruggiero, A. Ruiz-de-Angulo, E. Rezabal, J. C. Mareque-Rivas, X. Lopez, F. López-Gallego and L. Salassa, Chem. Sci., 2017, 8, 4619-4625.

12 S. Alonso-de Castro, A. L. Cortajarena, F. López-Gallego and L. Salassa, Angew. Chem., Int. Ed., 2018, 57, 3143-3147.

13 J. Gurruchaga-Pereda, V. Martínez-Martínez, E. Rezabal, X. Lopez, C. Garino, F. Mancin, A. L. Cortajarena and L. Salassa, ACS Catal., 2020, 10, 187-196.

14 L. Gong, Z. Lin, K. Harms and E. Meggers, Angew. Chem., Int. Ed., 2010, 7955-7957.

15 A. Ruffoni, F. Juliá, T. D. Svejstrup, A. J. McMillan, J. J. Douglas and D. Leonori, Nat. Chem., 2019, 11, 426-433.

16 S. Alonso-de Castro, A. Terenzi, S. Hager, B. Englinger, A. Faraone, J. C. Martínez, M. Galanski, B. K. Keppler, W. Berger and L. Salassa, Sci. Rep., 2018, 8, 17198.
17 J. Gurruchaga-Pereda, Á. Martínez, A. Terenzi and L. Salassa, Inorg. Chim. Acta, 2019, 495, 118981.

18 N. J. Farrer, J. A. Woods, L. Salassa, Y. Zhao, K. S. Robinson, G. Clarkson, F. S. Mackay and P. J. Sadler, Angew. Chem., Int. Ed., 2010, 49, 8905-8908.

19 D. J. Norman, A. Gambardella, A. R. Mount, A. F. Murray and M. Bradley, Angew. Chem., Int. Ed., 2019, 58, 14189-14192.

20 Z. Wang, N. Wang, S.-C. Cheng, K. Xu, Z. Deng, S. Chen, Z. Xu, K. Xie, M.-K. Tse, P. Shi, H. Hirao, C.-C. Ko and G. Zhu, Chemistry, 2019, 5, 3151-3165.

21 X. Liu, M. Atwater, J. Wang and Q. Huo, Colloids Surf., B, 2007, 58, 3-7.

22 S. S. Agasti, A. Chompoosor, C. You, P. Ghosh, C. K. Kim and V. M. Rotello, J. Am. Chem. Soc., 2009, 131, 5728-5729.

23 M. Diez-Castellnou, M. V. Salvia, S. Springhetti, F. Rastrelli and F. Mancin, Chem. - Eur. J., 2016, 22, 16957-16963.

$24 \mathrm{X}$. Yu and J. Zhang, Macrocyclic Polyamines, Wiley-VCH Verlag GmbH \& Co. KGaA, Weinheim, Germany, 2017, pp. 1-5.

25 A. Garaikoetxea Arguinzoniz, N. Gómez Blanco, P. Ansorena Legarra and J. C. Mareque-Rivas, Dalton Trans., 2015, 44, 7135-7138.

26 L. C. P. Gonçalves, H. R. Mansouri, E. L. Bastos, M. Abdellah, B. S. Fadiga, J. Sá, F. Rudroff and M. D. Mihovilovic, Catal. Sci. Technol., 2019, 9, 1365-1371.

27 B. Birdsall, R. W. King, M. R. Wheeler, C. A. Lewis, S. R. Goode, R. B. Dunlap and G. C. K. Roberts, Anal. Biochem., 1983, 132, 353-361.

28 M.-V. Salvia, F. Ramadori, S. Springhetti, M. Diez-Castellnou, B. Perrone, F. Rastrelli and F. Mancin, J. Am. Chem. Soc., 2015, 137, 886-892.

29 L. Gabrielli, D. Rosa-Gastaldo, M.-V. Salvia, S. Springhetti, F. Rastrelli and F. Mancin, Chem. Sci., 2018, 9, 4777-4784.

30 R. Tavano, D. Segat, E. Reddi, J. Kos, M. Rojnik, P. Kocbek, S. Iratni, D. Scheglmann, M. Colucci, I. M. R. Echevarria, F. Selvestrel, F. Mancin and E. Papini, Nanomedicine, 2010, 5, 881-896.

31 M. Diez-Castellnou, F. Mancin and P. Scrimin, J. Am. Chem. Soc., 2014, 136, 1158-1161.

32 S. Neri, S. Garcia Martin, C. Pezzato and L. J. Prins, J. Am. Chem. Soc., 2017, 139, 1794-1797.

33 F. della Sala, J. L.-Y. Chen, S. Ranallo, D. Badocco, P. Pastore, F. Ricci and L. J. Prins, Angew. Chem., Int. Ed., 2016, 55, 10737-10740. 